\title{
Research Article \\ Existence of Homoclinic Orbits for Hamiltonian Systems with Superquadratic Potentials
}

\author{
Jian Ding, Junxiang $X \mathbf{u}_{\text {, }}$ and Fubao Zhang \\ Department of Mathematics, Southeast University, Nanjing 210018, China \\ Correspondence should be addressed to Jian Ding, df2001101@126.com
}

Received 20 September 2009; Revised 19 November 2009; Accepted 6 December 2009

Recommended by Stephen Clark

This paper concerns solutions for the Hamiltonian system: $\dot{z}=2 H_{z}(t, z)$. Here $H(t, z)=(1 / 2) z$. $L z+W(t, z), L$ is a $2 N \times 2 N$ symmetric matrix, and $W \in C^{1}\left(\mathbb{R} \times \mathbb{R}^{2 N}, \mathbb{R}\right)$. We consider the case that $0 \in \sigma_{c}(-(2(d / d t)+L))$ and $W$ satisfies some superquadratic condition different from the type of Ambrosetti-Rabinowitz. We study this problem by virtue of some weak linking theorem recently developed and prove the existence of homoclinic orbits.

Copyright (C) 2009 Jian Ding et al. This is an open access article distributed under the Creative Commons Attribution License, which permits unrestricted use, distribution, and reproduction in any medium, provided the original work is properly cited.

\section{Introduction and the Main Results}

In this paper, we consider the existence of homoclinic orbits for the following Hamiltonian system:

$$
\dot{z}=2 H_{z}(t, z)
$$

where $H(t, z)=(1 / 2) z \cdot L z+W(t, z), L$ is a $2 N \times 2 N$ symmetric matrix-valued function, and $W \in C^{1}\left(\mathbb{R} \times \mathbb{R}^{2 N}, \mathbb{R}\right)$ is superquadratic both around 0 and at infinity in $z \in \mathbb{R}^{2 N}$.

A solution of (1.1) is called to be homoclinic to 0 if $z(t) \not \equiv 0$ and $z(t) \rightarrow 0$ as $|t| \rightarrow \infty$.

In recent years, the existence and multiplicity of homoclinic orbits for Hamiltonian systems have been investigated in many papers via variational methods. See, for example, [1-7] for the second-order systems and [8-12] for the first-order systems. We note that in most of the papers on the first order system (1.1) it was assumed that

$(\diamond) L$ is constant such that $\operatorname{sp}(\partial L) \cap i \mathbb{R}=\emptyset$, where $\operatorname{sp}(\partial L)$ denotes the set of all eigenvalues of $2 L$. 
Thus, if we let $\sigma(A)$ denote the spectrum of $A,(\diamond)$ means that $L$ is independent of $t$ and there is $\alpha>0$ such that $(-\alpha, \alpha) \cap \sigma(A)=\emptyset$. Consequently, the operator $A:=-(2(d / d t)+L)$ : $W^{1, p}\left(\mathbb{R}, \mathbb{R}^{2 N}\right) \rightarrow L^{p}\left(\mathbb{R}, \mathbb{R}^{2 N}\right)$ is a homeomorphism for all $p>1$. This is important for the variational arguments. Later in [13], Ding considered the case that $L$ depends periodically on $t$. He made assumptions on $L$ such that 0 lies in a gap of $\sigma(A)$. If additionally $W(t, z)$ is periodic in $t$ and satisfies some superquadratic or asymptotically quadratic conditions in $z$ at infinity, then infinitely many homoclinic orbits were obtained.

If $0 \in \sigma_{c}(A)$, then the problem is quite different in nature since the operator $A$ cannot lead the behavior at 0 of the equation. Ding and Willem considered this case in [14]. They assumed that

$\left(A_{0}\right) L(t) \in C\left(\mathbb{R}, \mathbb{R}^{4 N^{2}}\right)$ is 1-periodic. There exists $\alpha>0$ such that $(0, \alpha) \cap \sigma(A)=\emptyset$.

Under $\left(A_{0}\right), 0$ may belong to continuous spectrum of $A$. The authors managed to construct an appropriate Banach space, on which some embedding results necessary for variational arguments were obtained. Using a generalized linking theorem developed by Kryszewski and Szulkin in [15], they got one homoclinic orbit of (1.1). Later, Ding and Girardi obtained infinitely many homoclinic orbits in [16] under the conditions of [14] with an additional evenness assumption on $W$. Note that in both papers $W$ satisfies a condition of the type of Ambrosetti-Rabinowitz (see [17]), that is,

$$
\exists \mu>2 \text { such that } 0<\mu W(t, z) \leq W_{z}(t, z) z, \quad t \in \mathbb{R}, z \in \mathbb{R}^{2 N} \backslash\{0\} .
$$

The (A-R) condition is essential to prove the Palais-Smale condition since the variational functional $\Phi$ is strongly indefinite and $0 \in \sigma_{c}(-2(d / d t)+L)$. The argument of Palais-Smale condition is rather technical and not standard without the (A-R) condition. In this paper, we consider the existence of solutions of (1.1) under $\left(A_{0}\right)$ without the (A-R) condition on $W$.

We observed that just recently some abstract linking theorems were developed by Bartsch and Ding in [18]. These theorems are impactful to study the existence and multiplicity of solutions for the strongly indefinite problem. Many new results have been obtained by these theorems based on the use of $(C)_{c}$ sequence. See [19-21] for applications of these ideas. Note that in [19-21] 0 either is not a spectral point or is at most an isolated eigenvalue of finite multiplicity. Thus $(C)_{c}$ condition was checked by virtue of some very technical analysis. However, if $0 \in \sigma_{\mathcal{C}}(A)$, then we can find a sequence $\left\{z_{n}\right\} \subset H^{1}$ with $\left|z_{n}\right|_{L^{2}}=1$ and $\left|A z_{n}\right|_{L^{2}} \rightarrow 0$. Thus the operator $A$ cannot lead the behavior at 0 of the equation. Consequently, besides $(C)_{c}$ condition, it seems also hard to check the following condition necessary for the linking theorems in [19-21]:

$\left(\Phi_{1}\right)$ for any $c>0$, there exists $\zeta>0$ such that $\|z\|<\zeta\left\|P_{Y} z\right\|$ for all $z \in \Phi_{c}$.

Our work benefits from [14] and some weak linking theorem recently developed by Schechter and Zou in [22]. This theorem permits us first to study a sequence of approximating problems $\Phi_{\lambda}$ for $\lambda \in[1,2]$ (the initial problem corresponds to $\lambda=1$ ) for which a bounded Palais-Smale sequence of $\Phi_{\lambda}$ is given for almost each $\lambda \in[1,2]$. Then by monotonicity, we find a sequence of $\left\{\lambda_{n}\right\}$ and $\left\{w_{n}\right\}$ such that $\lambda_{n} \rightarrow 1, \Phi_{\lambda_{n}}^{\prime}\left(w_{n}\right)=0$, and $\Phi_{\lambda_{n}}\left(w_{n}\right) \leq d$. Since the sequence $\left\{w_{n}\right\}$ consists of critical points of $\Phi_{\lambda_{n}}$, then its boundedness can be checked. Consequently one solution of (1.1) is obtained. The idea of first studying approximating problems for which the existence of a bounded Palais-Smale sequence is given freely and then proving that the sequence of approximated critical points is bounded was originally introduced in [23]. See also [24]. 
We make the following assumptions.

$\left(A_{1}\right) W(t, z) \in C^{1}\left(\mathbb{R} \times \mathbb{R}^{2 N}, \mathbb{R}\right)$ is 1-periodic in $t . W(t, 0)=0$ for all $t \in \mathbb{R}$. There exist constants $c_{1}>0$ and $\mu>2$ such that $W_{z}(t, z) z \geq c_{1}|z|^{\mu}$ for $(t, z) \in \mathbb{R} \times \mathbb{R}^{2 N}$.

$\left(A_{2}\right)$ there exist $c_{2}, r>0$ such that $\left|W_{z}(t, z)\right| \leq c_{2}|z|^{\mu-1}$ for $t \in \mathbb{R}$ and $|z| \leq r$.

$\left(A_{3}\right)$ there exist $c_{3}, R \geq r$ and $p \geq \mu$ such that $\left|W_{z}(t, z)\right| \leq c_{3}|z|^{p-1}$ for $t \in \mathbb{R}$ and $|z| \geq R$.

$\left(A_{4}\right)$ there exists $b_{0}>2$ such that $\liminf _{z \rightarrow 0}\left(W_{z}(t, z) z / W(t, z)\right) \geq b_{0}$ uniformly for $t \in \mathbb{R}$;

$\left(A_{5}\right) \widetilde{W}(t, z):=(1 / 2) W_{z}(t, z) z-W(t, z)>0$ for all $t \in \mathbb{R}, z \in \mathbb{R}^{2 N} \backslash\{0\}$. There exist constants $b_{\infty}>0$ and $\beta>p(p-2) /(p-1)$ such that $\liminf _{|z| \rightarrow \infty} \widetilde{W}(t, z) /|z|^{\beta} \geq b_{\infty}$ uniformly for $t \in \mathbb{R}$.

Theorem 1.1. Let $\left(A_{0}\right),\left(A_{1}\right)-\left(A_{5}\right)$ be satisfied, then (1.1) has at least one homoclinic orbit.

Remark 1.2. We can easily check that the (A-R) condition implies $\left(A_{4}\right)$ and $\left(A_{5}\right)$. But the converse proposition is not true. See the following example:

$$
W(t, z)=|z|^{\mu}+(\mu-2)|z|^{\mu-\epsilon} \sin ^{2}\left(\frac{|z|^{\epsilon}}{\epsilon}\right)
$$

where $2<\mu<\infty, 0<\epsilon<\min \{\mu-2, \mu /(\mu-1)\}$ (see [25] or [26] for details).

If $W_{z}(t, z)=a|z|^{\mu-2} z+R_{z}(t, z), a>0, \mu \in(2, \infty)$ with $R$ satisfying

$\left(B_{1}\right) R \in C^{1}\left(\mathbb{R} \times \mathbb{R}^{2 N}, \mathbb{R}\right)$ is 1-periodic in $t$ and

$$
\begin{aligned}
& R_{z}(t, z)=o\left(|z|^{\mu-1}\right) \quad \text { as }|z| \longrightarrow 0, \\
& R_{z}(t, z)=o\left(|z|^{\mu-1}\right) \quad \text { as }|z| \longrightarrow \infty,
\end{aligned}
$$

uniformly in $t \in \mathbb{R}$, then

$\left(B_{2}\right) 0<R_{z}(t, z) z \leq(a(\mu-2) / 2)|z|^{\mu}$ for all $t \in \mathbb{R}, z \in \mathbb{R}^{2 N} \backslash\{0\}$.

Theorem 1.3. Let $\left(A_{0}\right),\left(B_{1}\right)$, and $\left(B_{2}\right)$ be satisfied, then (1.1) has at least one homoclinic orbit.

This paper is organized as follows. In Section 2 we will construct some appropriate variational space and obtain some embedding results necessary for our variational arguments. In Section 3 we will recall a weak linking theorem, by which we will give the proof of Theorems 1.1 and 1.3 in Section 4.

\section{Some Embedding Results}

In what follows, by $|\cdot|_{p}$ we denote the usual $L^{p}$-norm and by $(\cdot)_{2}$ the usual $L^{2}$-inner product. A standard Floquet reduction argument shows that $\sigma(A)=\sigma_{c}(A)$ (see [14]). 
Let $\{E(\lambda) ; \lambda \in \mathbb{R}\}$ be the spectral family of $A$. A possesses the polar decomposition $A=U|A|$ with $U=I-E(0)-E(-0)$. By $\left(A_{0}\right), 0$ is at most a continuous spectrum of $A$. $L^{2}$ has an orthogonal decomposition

$$
L^{2}=L^{2-} \oplus L^{2+},
$$

where $L^{2 \pm}:=\left\{u \in L^{2} ; U u= \pm u\right\}$.

Let $\Phi\left(|A|^{1 / 2}\right)$ denote the domain of $|A|^{1 / 2}$ and let $E$ be the space of the completion of $\Phi\left(|A|^{1 / 2}\right)$ under the norm

$$
\|u\|_{E}:=\left.\left.|| A\right|^{1 / 2} u\right|_{2}
$$

$E$ becomes a Hilbert space under the inner product

$$
(u, v)_{E}:=\left(|A|^{1 / 2} u,|A|^{1 / 2} v\right)_{2} .
$$

E possesses an orthogonal decomposition

$$
E=E^{-} \oplus E^{+},
$$

where $E^{ \pm} \supseteq L^{2 \pm} \cap \Phi\left(|A|^{1 / 2}\right)$.

Under $\left(A_{0}\right)$, it is easy to check

$$
E^{+}=L^{2+} \cap \boldsymbol{\Phi}\left(|A|^{1 / 2}\right), \quad\|\cdot\|_{E} \sim\|\cdot\|_{H^{1 / 2}} \text { on } E^{+} .
$$

Therefore, $E^{+}$can be embedded continuously into $L^{p}\left(\mathbb{R}, \mathbb{R}^{2 N}\right)$ for any $p \geq 2$ and compactly into $L_{\text {loc }}^{p}\left(\mathbb{R}, \mathbb{R}^{2 N}\right)$ for any $p \in[1, \infty)$.

For any $\epsilon>0$, set $L_{\epsilon}^{2-}:=E(-\epsilon) L^{2}$ and $E_{\epsilon}^{-}:=L_{\epsilon}^{2-} \cap \Phi\left(|A|^{1 / 2}\right)=L_{\epsilon}^{2-} \cap E^{-}$. Then on $E_{\epsilon}^{-}$, we also have $\|\cdot\|_{E} \sim\|\cdot\|_{H^{1 / 2}}$ and the same embedding conclusion as that of $E^{+}$.

Let $\widetilde{L}_{\epsilon}^{2-}:=L^{2-} \cap\left(\mathrm{cl}_{L^{2}}\left(\bigcup_{\lambda<-\epsilon} E(\lambda) L^{2}\right)\right)^{\perp}$ where $\mathrm{cl}_{L^{2}}(\cdot)$ stands for the closure of $\cdot$ in $L^{2}$.

For $\mu>2$, let $E_{\epsilon, \mu}^{-}$be the completion of $\widetilde{L}_{\epsilon}^{2-}$ under the norm

$$
\|u\|_{\mu}:=\left(\left.\left.|| A\right|^{1 / 2} u\right|_{2} ^{2}+|u|_{\mu}^{2}\right)^{1 / 2}
$$

and let $E_{\mu}^{-}$denote the completion of $\Phi(A) \cap L^{2-}$ with respect to the norm $\|\cdot\|_{\mu}$. Then $E_{\epsilon}^{-}$is a closed subspace of $E_{\mu}^{-}$, and $E_{\mu}^{-}$possesses the following decomposition:

$$
E_{\mu}^{-}=E_{\epsilon}^{-} \oplus E_{\epsilon, \mu}^{-}
$$

Moreover, $E_{\epsilon}^{-}$is orthogonal to $E_{\epsilon, \mu}^{-}$with respect to $(\cdot)_{E}$. true.

Let $E_{\mu}$ be the completion of $\Phi(A)$ under the norm $\|\cdot\|_{\mu}$. The following result holds 
Lemma 2.1 (see $[14])$. Under $\left(A_{0}\right), E_{\mu}$ has the direct sum decomposition

$$
E_{\mu}=E_{\mu}^{-} \oplus E^{+},
$$

and $E_{\mu}$ is embedded continuously in $L^{v}$ for any $v \in[\mu, \infty)$ and compactly in $L_{\text {loc }}^{v}$ for any $v \in[2, \infty)$.

\section{A Weak Linking Theorem}

In this section we state some weak linking theorem due to [22] which was first built in a Hilbert space. This theorem is still true in a reflexive Banach space (cf. Willem and Zou [25]).

Let $E$ be a reflexive Banach space with norm $\|\cdot\|$ and possess a direct sum decomposition $E=N \oplus M$, where $N \subset E$ is a closed and separable subspace. Since $N$ is separable, we can define a new norm $|z|_{w}$ satisfying $|z|_{w} \leq\|z\|$, for all $z \in N$ such that the topology induced by this norm is equivalent to the weak topology of $N$ on bounded subsets of $N$. For $z=v+w \in E$ with $v \in N$ and $w \in M$, we define $|z|_{w}^{2}=|v|_{w}^{2}+\|w\|^{2}$, then $|z|_{w} \leq\|z\|$, for all $z \in E$. In particular, if $z_{n}=v_{n}+w_{n}$ is $|\cdot|_{w}$-bounded and $z_{n} \rightarrow z$ under the norm $|\cdot|_{w}$ in $E$, then $v_{n} \rightarrow v$ weakly in $N, w_{n} \rightarrow w$ strongly in $M$, and $z_{n} \rightarrow v+w$ weakly in $E$. Let $Q \subset N$ be a $\|\cdot\|$-bounded open convex subset and let $p_{0} \in Q$ be a fixed point. Let $F$ be a $|\cdot|_{w}$-continuous map from $E$ onto $N$ satisfying the following.

(i) $\left.F\right|_{Q}=$ id; $F$ maps bounded sets to bounded sets.

(ii) there exists a fixed finite-dimensional subspace $E_{0}$ of $E$ such that $F(u-v)-(F(u)-$ $F(v)) \subset E_{0}$, for all $u, v \in E$.

(iii) $F$ maps finite-dimensional subspaces of $E$ into finite-dimensional subspaces of $E$.

Set $A:=\partial Q, B:=F^{-1}\left(p_{0}\right)$, where $\partial Q$ denotes the $\|\cdot\|$-boundary of $Q$. For $\Phi \in C^{1}(E, \mathbb{R})$, we introduce the class $\Gamma$ of mappings $h:[0,1] \times \bar{Q} \rightarrow E$ with the following properties.

(a) $h:[0,1] \times \bar{Q} \rightarrow E$ is $|\cdot|_{w}$-continuous.

(b) for any $\left(s_{0}, u_{0}\right) \in[0,1] \times \bar{Q}$, there is a $|\cdot|_{w}$-neighborhood $U_{\left(s_{0}, u_{0}\right)}$ such that $\{u-h(t, u)$ : $\left.(t, u) \in U_{\left(s_{0}, u_{0}\right)} \cap([0,1] \times \bar{Q})\right\} \subset E_{f i n}$, where $E_{f i n}$ denotes some finite-dimensional subspaces of $E$.

(c) $h(0, u)=u, \Phi(h(s, u)) \leq \Phi(u)$, for all $u \in \bar{Q}$.

The following is a variant weak linking theorem in [22].

Theorem 3.1. Let the family of $C^{1}$-functionals $\left(\Phi_{\curlywedge}\right)$ have the form

$$
\Phi_{\lambda}(u):=I(u)-\lambda J(u), \quad \forall \lambda \in[1,2] .
$$

Assume that the following conditions hold.

(1) $J(u) \geq 0$, for all $u \in E ; \Phi_{1}:=\Phi$.

(2) $I(u) \rightarrow \infty$ or $J(u) \rightarrow \infty$ as $\|u\| \rightarrow \infty$.

(3) $\Phi_{\lambda}$ is $|\cdot|_{w}$-upper semicontinuous; $\Phi_{\lambda}^{\prime}$ is weakly sequentially continuous on E. Moreover, $\Phi_{\lambda}$ maps bounded sets into bounded sets.

(4) $\sup _{A} \Phi_{\lambda}<\inf _{B} \Phi_{\lambda}$, for all $\lambda \in[1,2]$. 
Then for almost all $\lambda \in[1,2]$, there exists a sequence $\left\{u_{n}\right\}$ such that

$$
\sup _{n}\left\|u_{n}\right\|<\infty, \quad \Phi_{\lambda}^{\prime}\left(u_{n}\right) \longrightarrow 0, \quad \Phi_{\lambda}\left(u_{n}\right) \longrightarrow C_{\lambda}
$$

where $C_{\lambda}:=\inf _{h \in \Gamma} \sup _{u \in \bar{Q}} \Phi_{\lambda}(h(1, u)) \in\left[\inf _{B} \Phi_{\lambda}, \sup _{\bar{Q}} \Phi_{\lambda}\right]$.

Remark 3.2. Consider $E_{\mu}=E_{\mu}^{-} \oplus E^{+}$defined as in Section 2. Obviously, $E_{\mu}$ is reflexive. For $z_{0} \in E^{+}$with $\left\|z_{0}\right\|_{\mu}=1, E^{+}=\mathbb{R} z_{0} \oplus E_{1}^{+}$. Let $N=E_{\mu}^{-} \oplus \mathbb{R} z_{0}$ and $M=E_{1}^{+}$, then $E_{\mu}=N \oplus M$. It is easy to see that $N$ is a closed and separable subspace of $E_{\mu}$. For any $u \in E_{\mu}, u$ can be written as $u=u^{-}+s z_{0}+w^{+}$with $u^{-} \in E_{\mu}^{-}$and $w^{+} \in E_{1}^{+}$. For $R>0$, let $Q:=\{u:=$ $\left.u^{-}+s z_{0}, s>0, u^{-} \in E_{\mu}^{-},\|u\|_{\mu}<R\right\}$, then $p_{0}:=s_{0} z_{0} \in Q$ for $0<s_{0}<R$. Define $F: E_{\mu} \rightarrow N$ as $F u:=u^{-}+\left\|s z_{0}+w^{+}\right\|_{\mu} z_{0}$. Then it is easy to check that $F, Q$, and $p_{0}$ satisfy (i), (ii), and (iii). If we let $A:=\partial Q$ and $B:=F^{-1}\left(s_{0} z_{0}\right)=\left\{u:=s z_{0}+w^{+}, s \geq 0, w^{+} \in E_{1}^{+},\left\|s z_{0}+w^{+}\right\|_{\mu}=s_{0}\right\}$, then $A$ links $B$ (see Lemmas 4.2 and 4.3 in Section 4 ).

\section{The Proof of the Main Results}

Consider the functional

$$
\Phi(z):=\frac{1}{2}\left\|z^{+}\right\|_{E}^{2}-\frac{1}{2}\left\|z^{-}\right\|_{E}^{2}-\int_{\mathbb{R}} W(t, z),
$$

for $z=z^{+}+z^{-} \in E_{\mu}$. Then by assumptions $\left(A_{1}\right)-\left(A_{3}\right)$ and Lemma 2.1, $\Phi \in C^{1}\left(E_{\mu}, \mathbb{R}\right)$. A standard argument shows that any critical point of $\Phi$ is a homoclinic orbit of (1.1) (cf. [14]).

Set

$$
\Phi_{\lambda}(z):=\frac{1}{2}\left\|z^{+}\right\|_{E}^{2}-\lambda\left(\frac{1}{2}\left\|z^{-}\right\|_{E}^{2}+\int_{\mathbb{R}} W(t, z)\right)=I(z)-\lambda J(z), \quad \lambda \in[1,2] .
$$

Then $\Phi_{1}=\Phi$ and $J(z) \geq 0$. By $\left(A_{2}\right)$ and $\left(A_{3}\right)$,

$$
\left|W_{z}(t, z)\right| \leq C\left(|z|^{\mu-1}+|z|^{p-1}\right)
$$

where, as below, $C$ stands for some generic positive constant.

Together with $\left(A_{1}\right)$, one has

$$
\frac{c_{1}}{\mu}|z|^{\mu} \leq W(t, z) \leq C\left(|z|^{\mu}+|z|^{p}\right)
$$

Thus $I(z) \rightarrow \infty$ or $J(z) \rightarrow \infty$ if $\|z\|_{\mu}^{2}=\left\|z^{+}\right\|_{E}^{2}+\left\|z^{-}\right\|_{E}^{2}+|z|_{\mu}^{2} \rightarrow \infty$.

Lemma 4.1. $\Phi_{\lambda}$ is $|\cdot|_{w}$-upper semicontinuous and $\Phi_{\lambda}^{\prime}$ is weakly sequentially continuous.

Proof. For any $c \in \mathbb{R}$, assume that $z_{n} \in\left\{z \in E_{\mu}, \Phi_{\lambda}(z) \geq c\right\}$ with $z_{n} \rightarrow z$. Let $z_{n}=z_{n}^{+}+z_{n}^{-}$with $z_{n}^{+} \in E^{+}$and $z_{n}^{-} \in E_{\mu}^{-}$. Then $z_{n}^{+} \rightarrow z^{+}$in $E_{\mu}$ and hence sup $\left\|z_{n}^{+}\right\|<\infty$. Since $\Phi_{\lambda}\left(z_{n}\right) \geq c$ and $W\left(t, z_{n}\right) \geq 0$, we have sup $\left\|z_{n}^{-}\right\|<\infty$. 
By (4.4),

$$
C\left|z_{n}\right|_{\mu}^{\mu} \leq \int_{\mathbb{R}} W\left(t, z_{n}\right) \mathrm{d} t \leq \frac{1}{\lambda}\left(\frac{1}{2}\left\|z_{n}^{+}\right\|_{E}^{2}-\frac{\lambda}{2}\left\|z_{n}^{-}\right\|_{E}^{2}-c\right)<\infty
$$

Then sup $\left\|z_{n}\right\|_{\mu}<\infty$. By Lemma 2.1, $z_{n} \rightarrow z$ in $E_{\mu}, z_{n} \rightarrow z$ in $L_{\text {loc' }}^{\mu}$ and $z_{n}(t) \rightarrow z(t)$ a.e. for $t \in \mathbb{R}$. By Fatou's Lemma, $\Phi_{\lambda}(z) \geq c$. Therefore, $\Phi_{\lambda}$ is $|\cdot|_{w}$-upper semicontinuous.

Let $z_{n} \rightarrow z$ in $E_{\mu}$, then $z_{n} \rightarrow z$ in $L_{\mathrm{loc}^{\prime}}^{p}, 2 \leq p<\infty$. By (4.3), $W_{z}\left(t, z_{n}\right) \rightarrow W_{z}(t, z)$ in $L_{\mathrm{loc}}^{\mu /(\mu-1)}$ and $\int_{\mathbb{R}} W_{z}\left(t, z_{n}\right) v \rightarrow \int_{\mathbb{R}} W_{z}(t, z) v$ for any $v \in E_{\mu}$. Therefore, $\Phi_{\lambda}^{\prime}\left(z_{n}\right) \rightarrow \Phi_{\lambda}^{\prime}(z)$.

Lemma 4.2. There exist $b>0, r>0$ such that $\Phi_{\lambda}(z) \geq b>0$, for all $z \in E^{+}$with $\|z\|_{\mu}=r$, for all $\lambda \in[1,2]$.

Proof. By (4.4) and Lemma 2.1,

$$
\int_{\mathbb{R}} W(t, z) \leq C\left(|z|_{\mu}^{\mu}+|z|_{p}^{p}\right) \leq C\left(\|z\|_{\mu}^{\mu}+\|z\|_{\mu}^{p}\right)
$$

The conclusion is obvious.

Lemma 4.3. There exists $R>r>0$ such that $\left.\Phi_{\lambda}\right|_{\partial M}=0$ and $\sup _{M} \Phi_{\lambda}<d<\infty$ for all $\lambda \in[1,2]$, where $M:=\left\{z=x+s z_{0}, x \in E_{\mu}^{-},\|z\|_{\mu} \leq R, s>0\right\}$ and $z_{0} \in E^{+},\left\|z_{0}\right\|_{\mu}=1$.

Proof. For $z=x+s z_{0}$, by (4.4),

$$
\Phi_{\lambda}(z) \leq \frac{s^{2}}{2}\left\|z_{0}\right\|_{E}^{2}-\frac{1}{2}\|x\|_{E}^{2}-C \int_{\mathbb{R}}\left|x+s z_{0}\right|^{\mu}
$$

Since $E_{\mu}$ is continuously embedded in $L^{t}$ for $\mu \leq t<\infty$, there exists a continuous projection from $E_{\mu}^{-} \oplus \mathbb{R} z_{0}$ in $L^{\mu}$ to $\mathbb{R} z_{0}$. Thus, $\left|s z_{0}\right|_{\mu} \leq C\left|x+s z_{0}\right|_{\mu}$ for some $C>0$ and then

$$
\Phi_{\lambda}(z) \leq C s^{2}-C\|x\|_{E}^{2}-C s^{\mu}
$$

and thus the lemma follows easily.

Combining Lemmas 4.1-4.3 and Theorem 3.1, we get the following.

Lemma 4.4. Under $\left(A_{0}\right)$ and $\left(A_{1}\right)-\left(A_{3}\right)$, for almost every $\lambda \in[1,2]$, there exist $\left\{z_{n}\right\} \subseteq E_{\mu}$ such that

$$
\sup \left\|z_{n}\right\|_{\mu}<\infty, \quad \Phi_{\lambda}^{\prime}\left(z_{n}\right) \longrightarrow 0, \quad \Phi_{\lambda}\left(z_{n}\right) \longrightarrow C_{\lambda} \in[b, d]
$$

We need the following lemma which is a special case of a more general result due to Lions [27, 28]. 
Lemma 4.5. Let $a>0$ and $2 \leq p<\infty$. If $\left\{z_{n}\right\} \subset H^{1}$ is bounded and if

$$
\sup _{s \in \mathbb{R}} \int_{B(s, a)}\left|u_{n}\right|^{p} \longrightarrow 0, \quad n \longrightarrow \infty
$$

where $B(s, a):=(s-a, s+a)$, then $u_{n} \rightarrow 0$ in $L^{t}$ for $2<t<\infty$.

Lemma 4.6. Under $\left(A_{0}\right)-\left(A_{3}\right)$, let $\lambda \in[1,2]$ be fixed. For the sequence $\left\{z_{n}\right\}$ in Lemma 4.4, there exist $\left\{k_{n}\right\} \subset \mathbb{Z}$ such that, up to a subsequence, $u_{n}(t):=z_{n}\left(t+k_{n}\right)$ satisfies $u_{n} \rightarrow u_{\lambda} \neq 0, \Phi_{\lambda}^{\prime}\left(u_{\curlywedge}\right)=0$ and $\Phi_{\lambda}\left(u_{\curlywedge}\right) \leq d$.

Proof. Write $z_{n}=z_{n}^{+}+z_{n}^{-}$with $z_{n}^{+} \in E^{+}$and $z_{n}^{-} \in E_{\mu}^{-}$. Since sup $\left\|z_{n}\right\|_{\mu}<\infty$, sup $\left\|z_{n}^{+}\right\|_{E}<\infty$, let $A_{+}$denote the part of $A$ in $\Phi(A) \cap L^{2+}=H^{1} \cap L^{2+}:=H_{+}^{1}$. Then by $\left(A_{0}\right)$,

$$
A_{+}=\int_{\alpha}^{\infty} \lambda \mathrm{d} E(\lambda)
$$

Obviously, $A_{+}: H_{+}^{1} \subset L^{2+} \rightarrow L^{2+}$ has a bounded inverse $A_{+}^{-1}$. Since

$$
\begin{gathered}
\left|A_{+} z\right|_{2}^{2}=\int_{\alpha}^{\infty} \lambda|\mathrm{d} E(\lambda) z|_{2}^{2} \geq \alpha|z|_{2}^{2}, \\
|\dot{z}|_{2}=|A z+L z|_{2} \leq\left|A_{+} z\right|_{2}+|L z|_{2} \text { for } z \in H_{+}^{1},
\end{gathered}
$$

then we have

$$
\|z\|_{H^{1}} \leq C\left|A_{+} z\right|_{2} \text { for } z \in H_{+}^{1} \text {. }
$$

Set $v_{n}=A_{+}^{-1} z_{n}^{+} \in H_{+}^{1}$, then

$$
\left\|v_{n}\right\|_{H^{1}} \leq C\left|z_{n}^{+}\right|_{2} \leq C\left\|z_{n}^{+}\right\|_{E}<\infty
$$

We claim that $v_{n}$ is nonvanishing, that is, there exist $M>0, a>0$, and $y_{n} \in \mathbb{R}$ such that

$$
\liminf _{n \rightarrow \infty} \int_{B\left(y_{n}, a\right)}\left|v_{n}\right|^{2} \mathrm{~d} t \geq M
$$

Indeed, if not, by (4.14), $\left\{v_{n}\right\}$ is bounded in $H^{1}$. Lemma 4.5 shows that $v_{n} \rightarrow 0$ in $L^{t}$ for $2<t<\infty$. By (4.3),

$$
\begin{aligned}
\left|\int_{\mathbb{R}} W_{z}\left(t, z_{n}\right) v_{n}\right| & \leq C \int_{\mathbb{R}}\left(\left|z_{n}\right|^{\mu-1}+\left|z_{n}\right|^{p-1}\right)\left|v_{n}\right| \\
& \leq C\left(\left|z_{n}\right|_{\mu}^{\mu-1}\left|v_{n}\right|_{\mu}+\left|z_{n}\right|_{p}^{p-1}\left|v_{n}\right|_{p}\right) \\
& \longrightarrow 0 .
\end{aligned}
$$


Hence

$$
\left(z_{n}^{+}, v_{n}\right)_{E}=\Phi_{\lambda}^{\prime}\left(z_{n}\right) v_{n}+\lambda \int_{\mathbb{R}} W_{z}\left(t, z_{n}\right) v_{n} \longrightarrow 0
$$

Thus,

$$
\left|z_{n}^{+}\right|_{2}^{2}=\left(z_{n}^{+}, A_{+} v_{n}\right)_{L^{2}}=\left(z_{n}^{+}, v_{n}\right)_{E} \longrightarrow 0
$$

Therefore, for any $2 \leq t<\infty$,

$$
\left|z_{n}^{+}\right|_{t} \leq\left|z_{n}^{+}\right|_{2}^{1 / t}\left|z_{n}^{+}\right|_{2(t-1)}^{1-1 / t} \leq C\left\|z_{n}^{+}\right\|_{E}^{1-1 / t}\left|z_{n}^{+}\right|_{2}^{1 / t} \longrightarrow 0
$$

Thus we obtain

$$
\left\|z_{n}^{+}\right\|_{E}^{2}=\Phi_{\lambda}^{\prime}\left(z_{n}\right) z_{n}^{+}+\lambda \int_{\mathbb{R}} W_{z}\left(t, z_{n}\right) z_{n}^{+} \longrightarrow 0,
$$

and then

$$
\Phi_{\lambda}\left(z_{n}\right) \leq \frac{1}{2}\left\|z_{n}^{+}\right\|_{E}^{2} \longrightarrow 0,
$$

a contradiction. Choose $k_{n} \in \mathbb{Z}$ such that $\left|k_{n}-y_{n}\right|=\min \left\{\left|s-y_{n}\right|, s \in \mathbb{Z}\right\}$ and let $u_{n}:=k_{n} * z_{n}=$ $z_{n}\left(t+k_{n}\right):=u_{n}^{+}+u_{n}^{-}$. In view of the invariance of $E^{+}$under the action $*, u_{n}^{+}=k_{n} * z_{n}^{+} \in E^{+}$. Since $A$ commutes with $*$, then $A_{+}^{-1}$ also does. Therefore $\bar{v}_{n}:=k_{n} * v_{n}=A_{+}^{-1} u_{n}^{+}$. By (4.15),

$$
\left|\bar{v}_{n}\right|_{L^{2}(B(0, a+1))}^{2} \geq \frac{M}{2}
$$

Clearly,

$$
\left\|u_{n}\right\|_{\mu}=\left\|z_{n}\right\|_{\mu}<\infty
$$

Thus, up to a subsequence, we assume that

$$
u_{n} \rightarrow u_{\lambda} \quad \text { in } E_{\mu}, \quad u_{n} \longrightarrow u_{\curlywedge} \quad \text { in } L_{\text {loc }}^{t} \quad \text { for } t \geq 2 .
$$

We now establish that $u_{\lambda} \neq 0$. If not, $u_{n}^{+} \rightarrow 0$ in $L^{2}$, and then

$$
\left(\bar{v}_{n}, z\right)_{E}=\left(u_{n}^{+}, z\right)_{L^{2}} \longrightarrow 0 \quad \text { for all } z \in H^{1 / 2},
$$

which implies that

$$
\bar{v}_{n} \rightarrow 0 \quad \text { in } H^{1 / 2}, \quad \bar{v}_{n} \longrightarrow 0 \quad \text { in } L_{\text {loc }}^{t} \quad \text { for } t \geq 2,
$$


contradicting (4.22). By Lemma 4.1, $\Phi_{\lambda}^{\prime}$ is weakly continuous, hence we have

$$
\Phi_{\lambda}^{\prime}\left(u_{\lambda}\right)=\lim _{n \rightarrow \infty} \Phi_{\lambda}^{\prime}\left(u_{n}\right)=0
$$

By Fatou's Lemma, we obtain

$$
\begin{aligned}
\Phi_{\lambda}\left(u_{\curlywedge}\right) & =\Phi_{\lambda}\left(u_{\curlywedge}\right)-\frac{1}{2} \Phi_{\lambda}^{\prime}\left(u_{\curlywedge}\right) u_{\lambda} \\
& \leq \lim _{n \rightarrow \infty}\left(\Phi_{\lambda}\left(u_{n}\right)-\frac{1}{2} \Phi_{\lambda}^{\prime}\left(u_{n}\right) u_{n}\right) \\
& =\lim _{n \rightarrow \infty} \Phi_{\lambda}\left(u_{n}\right)=\lim _{n \rightarrow \infty} \Phi_{\lambda}\left(z_{n}\right) \\
& \leq d .
\end{aligned}
$$

As a straightforward consequence of Lemmas 4.4 and 4.6, we have the following.

Lemma 4.7. Under $\left(A_{0}\right)-\left(A_{3}\right)$, there exist $\left\{\lambda_{n}\right\} \subset[1,2],\left\{w_{n}\right\} \subset E_{\mu} \backslash\{0\}$ such that $\lambda_{n} \rightarrow$ $1, \Phi_{\lambda_{n}}^{\prime}\left(w_{n}\right)=0$, and $\Phi_{\lambda_{n}}\left(w_{n}\right) \leq d$.

Lemma 4.8. $\left\{w_{n}\right\}$ is bounded in $E_{\mu}$.

Proof. Our argument is motivated by [26]. Write $w_{n}=w_{n}^{+}+w_{n}^{-}$with $w_{n}^{+} \in E^{+}$and $w_{n}^{-} \in$ $E_{\mu}^{-}$. Since $\Phi_{\lambda_{n}}^{\prime}\left(w_{n}\right) w_{n}=0$, by $\left(A_{1}\right)$, then

$$
\left\|w_{n}^{+}\right\|_{E}^{2}-\lambda_{n}\left\|w_{n}^{-}\right\|_{E}^{2}=\lambda_{n} \int_{\mathbb{R}} W_{z}\left(t, w_{n}\right) w_{n} \geq C\left|w_{n}\right|_{\mu}^{\mu}
$$

Hence,

$$
\left|w_{n}\right|_{\mu}^{\mu} \leq C\left\|w_{n}^{+}\right\|_{E^{\prime}}^{2} \quad\left\|w_{n}^{-}\right\|_{E} \leq C\left\|w_{n}^{+}\right\|_{E^{\prime}}, \quad\left|w_{n}^{-}\right|_{\mu} \leq C\left\|w_{n}^{+}\right\|_{E}+C\left\|w_{n}^{+}\right\|_{E}^{2 / \mu}
$$

In the following, we show that $\left\|w_{n}^{+}\right\|_{E}$ is bounded. Choose $\epsilon_{0}>0$ small enough such that $b_{0}-\epsilon_{0}>2$. By $\left(A_{4}\right)$, there exists $0<r_{0} \leq 1$ such that

$$
W_{z}(t, z) z \geq\left(b_{0}-\epsilon_{0}\right) W(t, z)
$$

for all $t \in \mathbb{R}$ and $|z| \leq r_{0}$. By $\left(A_{3}\right)$ and $\left(A_{5}\right)$, for all $t \in \mathbb{R}$ and $|z| \geq r_{0}$, we can choose $C, C^{\prime}>0$ such that

$$
\begin{gathered}
\left|W_{z}(t, z)\right| \leq C|z|^{p-1}, \\
W_{z}(t, z) z-2 W(t, z) \geq C^{\prime}|z|^{\beta} .
\end{gathered}
$$


Since $\Phi_{\lambda_{n}}\left(w_{n}\right) \leq d$ and $\Phi_{\lambda_{n}}^{\prime}\left(w_{n}\right)=0$, then we have

$$
\left(\frac{1}{2}-\frac{1}{b_{0}-\epsilon_{0}}\right)\left(\left\|w_{n}^{+}\right\|_{E}^{2}-\lambda_{n}\left\|w_{n}^{-}\right\|_{E}^{2}\right)+\lambda_{n} \int_{\mathbb{R}}\left(\frac{1}{b_{0}-\epsilon_{0}} W_{z}\left(t, w_{n}\right) w_{n}-W\left(t, w_{n}\right)\right) \leq d
$$

Thus, by (4.31), (4.32), and $\left(A_{5}\right)$, we obtain

$$
\begin{aligned}
\left\|w_{n}^{+}\right\|_{E}^{2}-\lambda_{n}\left\|w_{n}^{-}\right\|_{E}^{2} & \leq C\left(\int_{\left|w_{n}\right|<r_{0}}+\int_{\left|w_{n}\right| \geq r_{0}}\right)\left(W\left(t, w_{n}\right)-\frac{1}{b_{0}-\epsilon_{0}} W_{z}\left(t, w_{n}\right) w_{n}\right) \mathrm{d} t+C \\
& \leq C \int_{\left|w_{n}\right| \geq r_{0}}\left(W\left(t, w_{n}\right)-\frac{1}{b_{0}-\epsilon_{0}} W_{z}\left(t, w_{n}\right) w_{n}\right) \mathrm{d} t+C \\
& \leq C \int_{\left|w_{n}\right| \geq r_{0}} W_{z}\left(t, w_{n}\right) w_{n} \mathrm{~d} t+C \\
& \leq C \int_{\left|w_{n}\right| \geq r_{0}}\left|w_{n}\right|^{p} \mathrm{~d} t+C .
\end{aligned}
$$

By $(4.33)$ and $\left(A_{5}\right)$,

$$
\begin{aligned}
C & \geq \Phi_{\lambda_{n}}\left(w_{n}\right)-\frac{1}{2} \Phi_{\lambda_{n}}^{\prime}\left(w_{n}\right) w_{n} \\
& =\int_{\mathbb{R}}\left(\frac{1}{2} W_{z}\left(t, w_{n}\right) w_{n}-W\left(t, w_{n}\right)\right) \mathrm{d} t \\
& \geq C \int_{\left|w_{n}\right| \geq r_{0}}\left|w_{n}\right|^{\beta} \mathrm{d} t .
\end{aligned}
$$

Choose $v>p$ sufficiently large such that $(v p(p-2)) /(v(p-1)-p)<\beta$. Let $t:=v(p-\beta) /(v-$ $\beta) p$, then by $\left(A_{5}\right), 0<t<1 /(p-1)$ for $v$ being large enough. By Hölder's inequality and Lemma 2.1, we have

$$
\begin{aligned}
\int_{\left|w_{n}\right| \geq r_{0}}\left|w_{n}\right|^{p} \mathrm{~d} t & \leq\left(\int_{\left|w_{n}\right| \geq r_{0}}\left|w_{n}\right|^{\beta}\right)^{(1-t) p / \beta}\left(\int_{\mathbb{R}}\left|w_{n}\right|^{\nu}\right)^{t p / v} \\
& \leq C\left|w_{n}\right|_{v}^{t p} \\
& \leq C\left(\left\|w_{n}^{+}\right\|_{E}+\left\|w_{n}^{-}\right\|_{E}+\left|w_{n}\right|_{\mu}\right)^{t p} \\
& \leq C\left(\left\|w_{n}^{+}\right\|_{E}+\left\|w_{n}^{+}\right\|_{E}^{2 / \mu}\right)^{t p} \\
& \leq C\left(\left\|w_{n}^{+}\right\|_{E}^{t p}+\left\|w_{n}^{+}\right\|_{E}^{2 t p / \mu}\right) .
\end{aligned}
$$


By (4.29), (4.35), and (4.37),

$$
\int_{\mathbb{R}}\left|w_{n}\right|^{\mu} \leq C\left(\left\|w_{n}^{+}\right\|_{E}^{t p}+\left\|w_{n}^{+}\right\|_{E}^{2 t p / \mu}+1\right)
$$

and then

$$
\left|w_{n}\right|_{\mu} \leq C\left(\left\|w_{n}^{+}\right\|_{E}^{t p / \mu}+\left\|w_{n}^{+}\right\|_{E}^{2 t p / \mu^{2}}+1\right)
$$

Using (4.3), (4.37), and (4.39), from $\Phi_{\lambda_{n}}^{\prime}\left(w_{n}\right) w_{n}^{+}=0$, we obtain

$$
\begin{aligned}
\left\|w_{n}^{+}\right\|_{E}^{2} & \leq C \int_{\mathbb{R}}\left(\left|w_{n}\right|^{\mu-1}\left|w_{n}^{+}\right|+\left|w_{n}\right|^{p-1}\left|w_{n}^{+}\right|\right) \mathrm{d} t \\
& \leq C\left(\left|w_{n}\right|_{\mu}^{\mu-1}+\left(\int_{\mathbb{R}}\left|w_{n}\right|^{p}\right)^{(p-1) / p}\right)\left\|w_{n}^{+}\right\|_{E} \\
& \leq C\left(\left|w_{n}\right|_{\mu}^{\mu-1}+\left(\int_{\left\{t|| w_{n}(t) \mid \leq r_{0}\right\}}\left|w_{n}\right|^{p}+\int_{\left\{t|| w_{n}(t) \mid \geq r_{0}\right\}}\left|w_{n}\right|^{p}\right)^{(p-1) / p}\right)\left\|w_{n}^{+}\right\|_{E} \\
& \leq C\left(\left|w_{n}\right|_{\mu}^{\mu-1}+\left(\int_{\mathbb{R}}\left|w_{n}\right|^{\mu}\right)^{(p-1) / p}+\left(\int_{\left\{t|| w_{n}(t) \mid \geq r_{0}\right\}}\left|w_{n}\right|^{p}\right)^{(p-1) / p}\right)\left\|w_{n}^{+}\right\|_{E} \\
& \leq C\left(\left|w_{n}\right|_{\mu}^{\mu-1}+\left|w_{n}\right|_{\mu}^{\mu(p-1) / p}+\left\|w_{n}^{+}\right\|_{E}^{t(p-1)}+\left\|w_{n}^{+}\right\|_{E}^{2 t(p-1) / \mu}\right)\left\|w_{n}^{+}\right\|_{E} \\
& \leq C\left(\left\|w_{n}^{+}\right\|_{E}^{t p(\mu-1) / \mu}+\left\|w_{n}^{+}\right\|_{E}^{2 t p(\mu-1) / \mu^{2}}+\left\|w_{n}^{+}\right\|_{E}^{t(p-1)}+\left\|w_{n}^{+}\right\|_{E}^{2 t(p-1) / \mu}+1\right)\left\|w_{n}^{+}\right\|_{E},
\end{aligned}
$$

which implies sup $\left\|w_{n}^{+}\right\|_{E}<\infty$ since $t(p-1)<1$.

Proof of Theorem 1.1. Since $\left\{w_{n}\right\}$ is bounded, $w_{n} \rightarrow w$ in $E_{\mu}$ and $w_{n} \rightarrow w$ in $L_{\mathrm{loc}}^{t}$ for $2 \leq t<\infty$. We show that $w \neq 0$.

In fact, by (4.3) and (4.30),

$$
\begin{aligned}
\left|\int_{\mathbb{R}} W_{z}\left(t, w_{n}\right) w_{n}^{+}\right| & \leq C \int_{\mathbb{R}}\left(\left|w_{n}\right|^{\mu-1}+\left|w_{n}\right|^{p-1}\right)\left|w_{n}^{+}\right| \\
& \leq C\left(\left|w_{n}\right|_{\mu}^{\mu-1}+\left|w_{n}\right|_{p}^{p-1}\right)\left\|w_{n}^{+}\right\|_{E} \\
& \leq C\left(\left\|w_{n}\right\|_{\mu}^{\mu-1}+\left\|w_{n}\right\|_{\mu}^{p-1}\right)\left\|w_{n}^{+}\right\|_{E} \\
& \leq C\left(\left\|w_{n}^{+}\right\|_{E}^{\mu}+\left\|w_{n}^{+}\right\|_{E}^{2(\mu-1) / \mu+1}+\left\|w_{n}^{+}\right\|_{E}^{p}+\left\|w_{n}^{+}\right\|_{E}^{2(p-1) / \mu+1}\right) .
\end{aligned}
$$


It follows from $\Phi_{\lambda_{n}}^{\prime}\left(w_{n}\right) w_{n}^{+}=0$ that

$$
\begin{aligned}
\left\|w_{n}^{+}\right\|_{E}^{2} & =\lambda_{n} \int_{\mathbb{R}} W_{z}\left(t, w_{n}\right) w_{n}^{+} \\
& \leq C\left(\left\|w_{n}^{+}\right\|_{E}^{\mu}+\left\|w_{n}^{+}\right\|_{E}^{2(\mu-1) / \mu+1}+\left\|w_{n}^{+}\right\|_{E}^{p}+\left\|w_{n}^{+}\right\|_{E}^{2(p-1) / \mu+1}\right),
\end{aligned}
$$

which implies that there exists $C_{0}>0$ such that $\left\|w_{n}^{+}\right\|_{E} \geq C_{0}$.

If $\left\{w_{n}^{+}\right\}$is vanishing, then

$$
\left\|w_{n}^{+}\right\|_{E}^{2}=\lambda_{n} \int_{\mathbb{R}} W_{z}\left(t, w_{n}\right) w_{n}^{+} \longrightarrow 0,
$$

a contradiction. Hence $\left\{w_{n}^{+}\right\}$is nonvanishing.

Just along the proof of Lemma 4.6, we can see that there exist $M>0$ and $a>0$ such that

$$
\int_{B(0, a+1)}\left|\bar{w}_{n}^{+}\right| \mathrm{d} t \geq \frac{M}{2}
$$

where $\bar{w}_{n}^{+}:=w_{n}^{+}\left(t+y_{n}\right)$.

Set $\bar{w}_{n}^{-}:=w_{n}^{-}\left(t+y_{n}\right)$ and $\bar{w}_{n}=\bar{w}_{n}^{+}+\bar{w}_{n}^{-}$. Then sup $\left\|\bar{w}_{n}\right\|_{\mu}<\infty$ and then $\bar{w}_{n} \rightarrow \bar{w}$, $\bar{w}_{n}^{+} \rightarrow \bar{w}^{+}$, and $\bar{w}_{n}^{-} \rightarrow \bar{w}^{-}$. By Lemma $2.1, \bar{w}_{n}^{+} \rightarrow \bar{w}^{+}$in $L_{\text {loc' }}^{2}$ and hence

$$
\int_{B(0, a+1)}\left|\bar{w}^{+}\right|^{2} \geq \frac{M}{2}>0
$$

It follows that $\bar{w} \neq 0$.

Since $\Phi_{\lambda_{n}}^{\prime}\left(\bar{w}_{n}\right)=0$, using Lebesgue's theorem, then we obtain

$$
\begin{aligned}
-\Phi^{\prime}(\bar{w}) \phi= & \Phi_{\lambda_{n}}^{\prime}\left(\bar{w}_{n}\right) \phi-\Phi_{\lambda_{n}}^{\prime}(\bar{w}) \phi+\Phi_{\lambda_{n}}^{\prime}(\bar{w}) \phi-\Phi^{\prime}(\bar{w}) \phi \\
= & \left\langle\bar{w}_{n}^{+}-\bar{w}^{+}, \phi\right\rangle_{E}-\lambda_{n}\left\langle\bar{w}_{n}^{-}-\bar{w}^{-}, \phi\right\rangle_{E}-\lambda_{n} \int_{\mathbb{R}}\left(W_{z}\left(t, \bar{w}_{n}\right)-W_{z}(t, \bar{w})\right) \phi \\
& +\left(1-\lambda_{n}\right)\langle\bar{w}, \phi\rangle_{E}+\left(1-\lambda_{n}\right) \int_{\mathbb{R}} W_{z}(t, \bar{w}) \phi \longrightarrow 0
\end{aligned}
$$

for any $\phi \in C_{0}^{\infty}$, that is, $\Phi^{\prime}(\bar{w})=0$.

Proof of Theorem 1.3. It is easy to check that $W_{z}(t, z)=a|z|^{\mu-2} z+R_{z}(t, z)$ satisfies all the assumptions of Theorem 1.1 with $b_{0}=\beta=\mu$.

\section{Acknowledgments}

The authors would like to thank the reviewers for the valuable suggestions. This work was supported by the Natural Science Foundation of China. 


\section{References}

[1] A. Ambrosetti and V. Coti-Zelati, "Multiplicité des orbites homoclines pour des systèmes conservatifs," Comptes Rendus de l'Académie des Sciences. Série I, vol. 314, no. 8, pp. 601-604, 1992.

[2] V. Coti-Zelati and P. H. Rabinowitz, "Homoclinic orbits for second order Hamiltonian systems possessing superquadratic potentials," Journal of the American Mathematical Society, vol. 4, no. 4, pp. 693-727, 1991.

[3] Y. H. Ding, "Existence and multiplicity results for homoclinic solutions to a class of Hamiltonian systems," Nonlinear Analysis: Theory, Methods \& Applications, vol. 25, no. 11, pp. 1095-1113, 1995.

[4] Y. H. Ding and M. Girardi, "Periodic and homoclinic solutions to a class of Hamiltonian systems with the potentials changing sign," Dynamic Systems and Applications, vol. 2, no. 1, pp. 131-145, 1993.

[5] W. Omana and M. Willem, "Homoclinic orbits for a class of Hamiltonian systems," Differential and Integral Equations, vol. 5, no. 5, pp. 1115-1120, 1992.

[6] P. H. Rabinowitz, "Homoclinic orbits for a class of Hamiltonian systems," Proceedings of the Royal Society of Edinburgh A, vol. 114, no. 1-2, pp. 33-38, 1990.

[7] P. H. Rabinowitz and K. Tanaka, "Some results on connecting orbits for a class of Hamiltonian systems," Mathematische Zeitschrift, vol. 206, no. 3, pp. 473-499, 1991.

[8] V. Coti-Zelati, I. Ekeland, and É. Séré, "A variational approach to homoclinic orbits in Hamiltonian systems," Mathematische Annalen, vol. 288, no. 1, pp. 133-160, 1990.

[9] Y. H. Ding and S. J. Li, "Homoclinic orbits for first order Hamiltonian systems," Journal of Mathematical Analysis and Applications, vol. 189, no. 2, pp. 585-601, 1995.

[10] H. Hofer and K. Wysocki, "First-order elliptic systems and the existence of homoclinic orbits in Hamiltonian systems," Mathematische Annalen, vol. 288, no. 3, pp. 483-503, 1990.

[11] É. Séré, "Existence of infinitely many homoclinic orbits in Hamiltonian systems," Mathematische Zeitschrift, vol. 209, no. 1, pp. 27-42, 1992.

[12] K. Tanaka, "Homoclinic orbits in a first order superquadratic Hamiltonian system: convergence of subharmonic orbits," Journal of Differential Equations, vol. 94, no. 2, pp. 315-339, 1991.

[13] Y. H. Ding, "Multiple homoclinics in a Hamiltonian system with asymptotically or super linear terms," Communications in Contemporary Mathematics, vol. 8, no. 4, pp. 453-480, 2006.

[14] Y. H. Ding and M. Willem, "Homoclinic orbits of a Hamiltonian system," Zeitschrift für Angewandte Mathematik und Physik, vol. 50, no. 5, pp. 759-778, 1999.

[15] W. Kryszewski and A. Szulkin, "Generalized linking theorem with an application to a semilinear Schrödinger equation," Advances in Differential Equations, vol. 3, no. 3, pp. 441-472, 1998.

[16] Y. H. Ding and M. Girardi, "Infinitely many homoclinic orbits of a Hamiltonian system with symmetry," Nonlinear Analysis: Theory, Methods E Applications, vol. 38, no. 3, pp. 391-415, 1999.

[17] A. Ambrosetti and P. H. Rabinowitz, "Dual variational methods in critical point theory and applications," Journal of Functional Analysis, vol. 14, pp. 349-381, 1973.

[18] T. Bartsch and Y. H. Ding, "Deformation theorems on non-metrizable vector spaces and applications to critical point theory," Mathematische Nachrichten, vol. 279, no. 12, pp. 1267-1288, 2006.

[19] Y. H. Ding and L. Jeanjean, "Homoclinic orbits for a nonperiodic Hamiltonian system," Journal of Differential Equations, vol. 237, no. 2, pp. 473-490, 2007.

[20] T. Bartsch and Y. H. Ding, "Solutions of nonlinear Dirac equations," Journal of Differential Equations, vol. 226, no. 1, pp. 210-249, 2006.

[21] Y. H. Ding and B. Ruf, "Solutions of a nonlinear dirac equation with external fields," Archive for Rational Mechanics and Analysis, vol. 190, no. 1, pp. 57-82, 2008.

[22] M. Schechter and W. Zou, “Weak linking theorems and Schrödinger equations with critical Sobolev exponent," Control, Optimisation and Calculus of Variations, vol. 9, pp. 601-619, 2003.

[23] L. Jeanjean, "On the existence of bounded Palais-Smale sequences and application to a LandesmanLazer-type problem set on $\mathbb{R}^{N}$," Proceedings of the Royal Society of Edinburgh A, vol. 129, no. 4, pp. 787-809, 1999.

[24] L. Jeanjean and K. Tanaka, “A positive solution for a nonlinear Schrödinger equation on $\mathbb{R}^{N}$," Indiana University Mathematics Journal, vol. 54, no. 2, pp. 443-464, 2005.

[25] M. Willem and W. Zou, "On a semilinear Dirichlet problem and a nonlinear Schrödinger equation with periodic potential," Indiana University Mathematics Journal, vol. 52, pp. 109-132, 2003. 
[26] W. Zou and M. Schechter, Critical Point Theory and Its Applications, Springer, New York, NY, USA, 2006.

[27] P. L. Lions, "The concentration-compactness principle in the calculus of variations. The locally compact case. Part I," Annales de l'Institut Henri Poincaré C, vol. 1, pp. 109-145, 1984.

[28] P. L. Lions, "The concentration-compactness principle in the calculus of variations. The locally compact case. Part II," Annales de l'Institut Henri Poincaré C, vol. 1, pp. 223-283, 1984. 


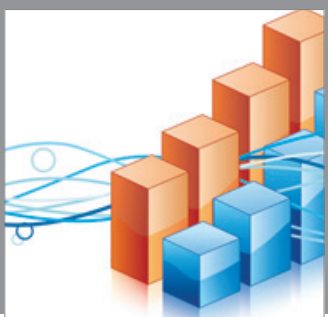

Advances in

Operations Research

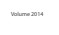

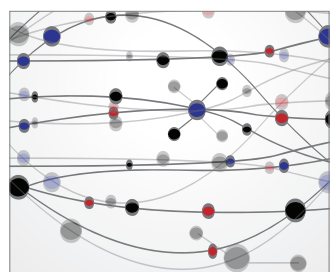

\section{The Scientific} World Journal
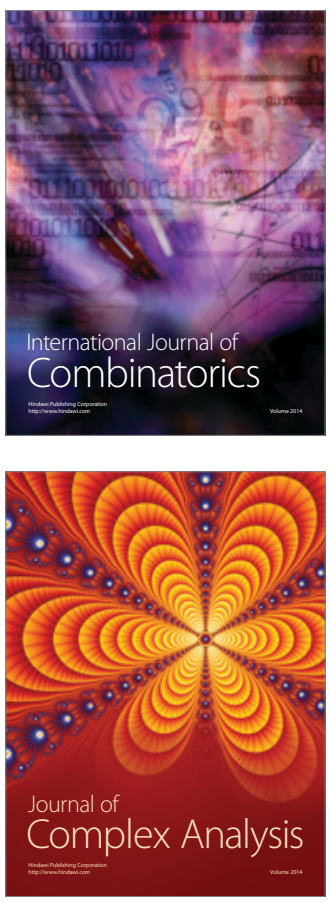

International Journal of

Mathematics and

Mathematical

Sciences
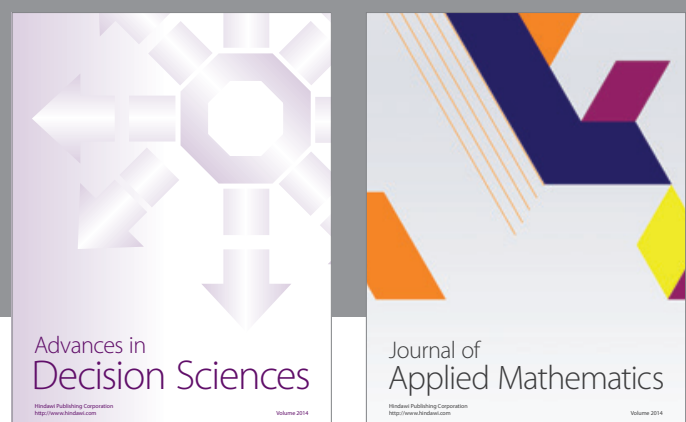

Journal of

Applied Mathematics
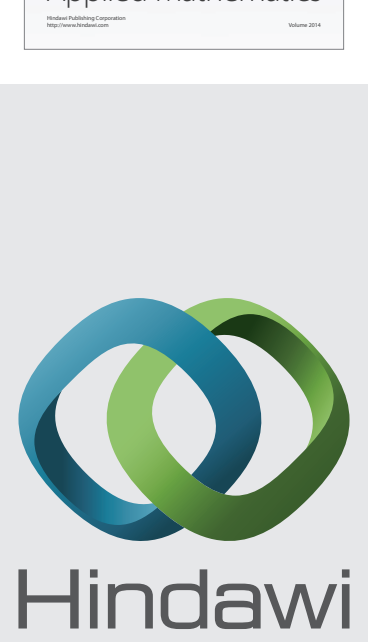

Submit your manuscripts at http://www.hindawi.com
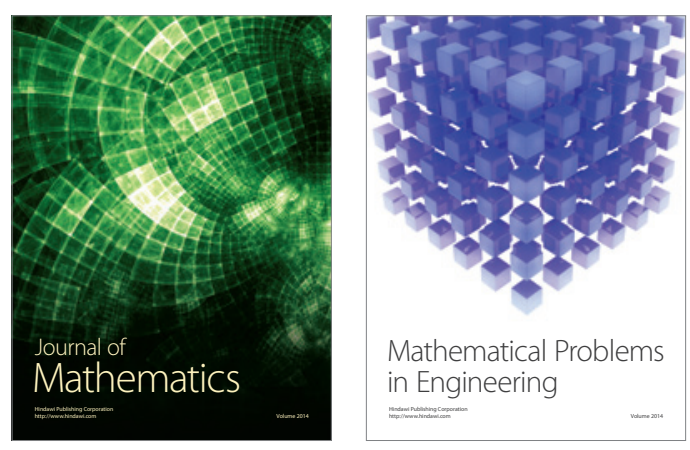

Mathematical Problems in Engineering
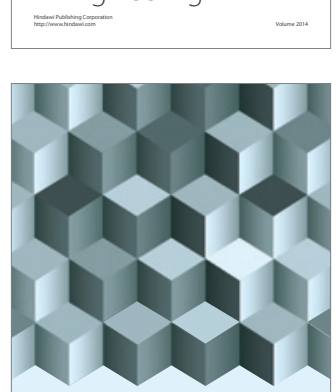

Journal of

Function Spaces
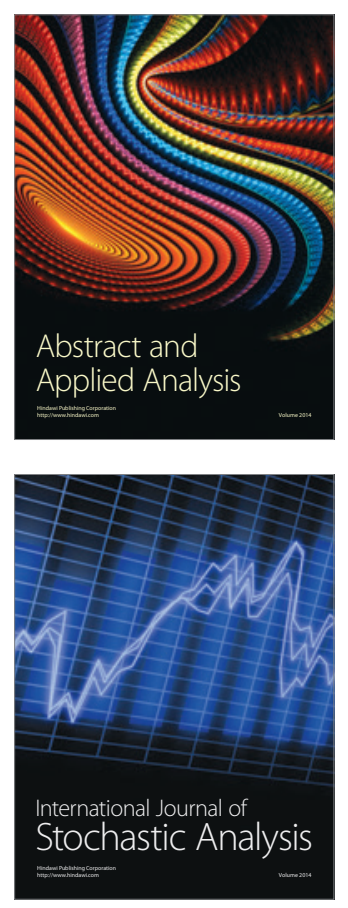

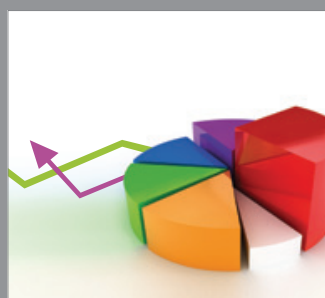

ournal of

Probability and Statistics

Promensencen
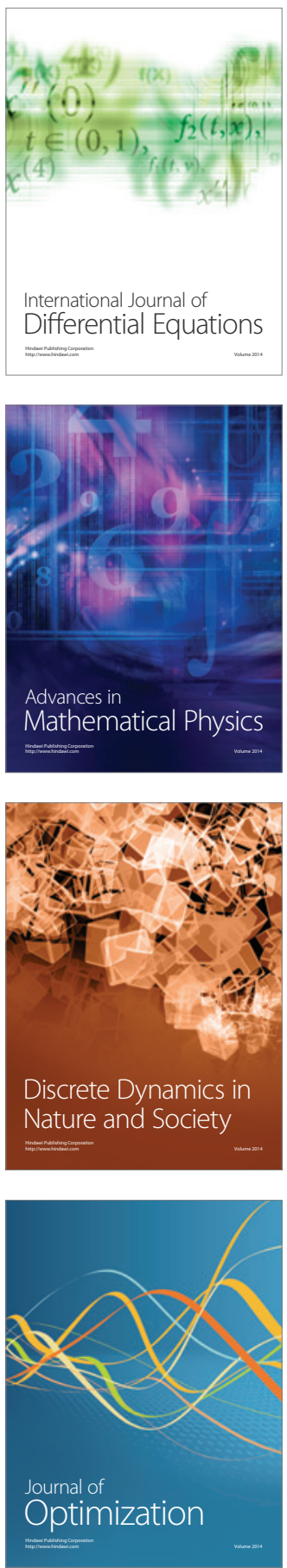